\title{
A Study on Compression Techniques for off-the-person Electrocardiogram Signals
}

\author{
António Cerca ${ }^{1} \quad$ André Lourenço ${ }^{1,2} \quad$ Artur Ferreira $^{1}$ \\ ${ }^{1}$ Instituto Superior de Engenharia de Lisboa, ${ }^{2}$ CardioID Technologies
}

\begin{abstract}
The compression of Electrocardiography (ECG) signals acquired in off-the-person scenarios requires methods that cope with noise and other impairments on the acquisition process. In this paper, after a brief review of common onthe-person ECG signal compression algorithms, we propose and evaluate techniques for this compression task with offthe-person acquired signals, in both lossy and lossless scenarios, evaluated with standard metrics. Our experimental results show that the joint use of Linear Predictive Coding and LempelZiv-Welch is an adequate lossless approach, and the amplitude scaling followed by the Discrete Wavelet Transform achieves the best compression ratio, with a small distortion, among the lossy techniques.
\end{abstract}

\section{INTRODUCTION}

Electrocardiography (ECG) is the process of recording the electrical activity of the heart [10]. It is widely used in medicine since it can alert for a variety of heart diseases. Recently, the demand for portable and accurate ECG monitoring has grown substantially, with ECG signals acquired in an off-the-person procedure using dry-electrodes embedded on everyday-use objects [1]. Besides medicine, the ECG signal holds relevant information for other applications, e.g. fatigue monitoring and biometrics. The dissemination of offthe-person ECG monitoring systems leads to the need of signal processing methodologies and the use of compression techniques, for storage and transmission purposes.

This paper addresses the task of compression of offthe-person ECG signals acquired using dry electrodes [1], which is a much more challenging task than the well-known typical use of gelled electrodes for clinical applications. The remainder of this paper is organized as follows: Section II presents the off-the-person ECG signals and the setup for their acquisition; Section III reviews data compression techniques for ECG signals; on Section IV, we address the experimental evaluation of the proposed techniques, with standard metrics. Section V ends the paper with some remarks and directions for future work.

\section{OFF-THE-PERSON ECG SIGNALS}

The ECG is the electrical signal emitted by the heart on the successive contractions and distensions of the heart muscle, named myocardium [10]. The usual way to acquire ECG signals uses electrodes directly attached to the body surface. It uses a gel or a conductive paste that enables the proper physical contact with the skin and an adequate capture of the cardiac signal. These clinical methods may require the placement of, for example, up to twelve electrodes on the surface of the body to extract a good ECG signal [1] and

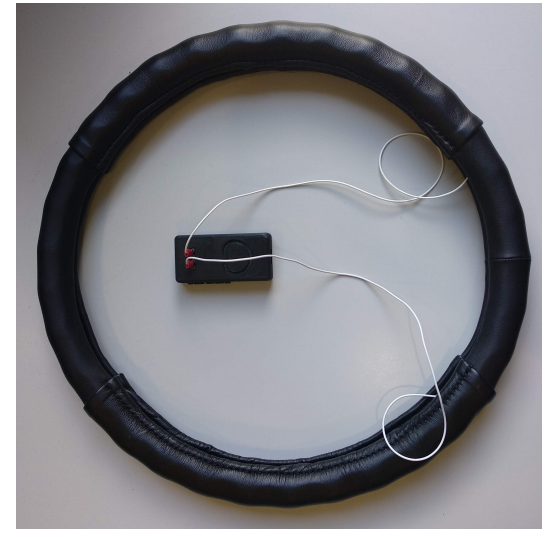

Fig. 1. CardioWheel [6]: a steering wheel cover with conductive leather connected with box containing embedded electronics.

are limited to a restricted physical space of use, such as an ambulance or a treatment room.

Off-the-person ECG techniques aim to acquire ECG signals in a less intrusive way using hands as contact points, allowing the acquisition of signals without sensors placed on the body, but rather in objects of everyday use [1]. The purpose of these methods is to make the acquisition of signals almost involuntarily, without impact on the person's daily actions. The components used in this method are called dryelectrodes as they do not require the use of any conductive gels or pastes, using human perspiration to a better contact with the person's skin. These electrodes can be placed on any equipment, such as, for instance, computer mice, keyboards, mobile phones, watches and cars's steering wheels, as depicted in Figure 1. To achieve an acceptable biometric signal, only two electrodes are required. However, the method is more sensitive to noise, as compared to the on-the-person ECG acquisition methods, leading to the need of signal processing tasks on the signal. After signal processing task, the main signal characteristics are preserved, enabling some analysis similar to those achieved with hospitalar systems, such as heartbeating detection.

Figure 2 presents a comparison of the signals acquired on the chest with the CardioWheel device, that acquires off-theperson ECG signals. In both cases, we identify the types of wave $\mathrm{P}, \mathrm{Q}, \mathrm{R}, \mathrm{S}$, and T, but with more noise on the off-theperson signal. The medical analysis of an ECG signal focuses mainly on the QRS wave complex. However, the P and T waves also have a high clinical value. Cardiac abnormalities are detected by considering the mean amplitude of each wave 


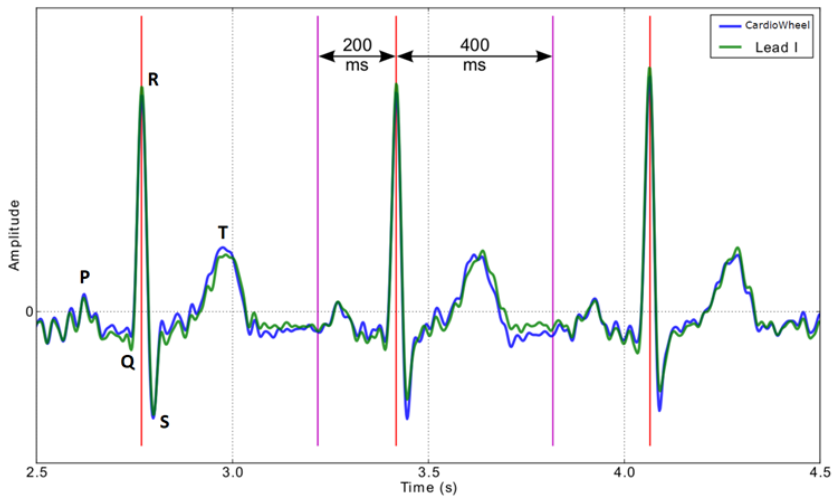

Fig. 2. Comparison between off-the-person (blue line) signal with on-theperson ECG (green line) signal. The PQRST complex.

as well as the time intervals between them. Typically, the signal voltage values may range from 1 to $10 \mathrm{mV}$, with signal frequency values ranging from 0.05 to $100 \mathrm{~Hz}$ and a heart rate oscillating from 60 to 100 beats per minute [8].

\section{DATA COMPRESSION TECHNIQUES}

Data compression is the process of encoding information using fewer bits than the original representation. It can be either lossless or lossy. In the lossless approach, also named compaction, the decoded signal is exactly the same as the original signal. On the lossy approach, the decoded signal is similar to the original version, thus it has some distortion. The lossy approaches achieve much higher compression ratios than the lossless techniques, at the expense of distortion, which is established by the lossy encoder.

The ECG signal acquisition produces a sequence of numbers recording the amplitude of the signal, which is named as a digital signal. This sequence of numbers when displayed as a graph, yields a representation similar to those depicted in Figure 2. Thus, for the compression tasks of ECG signals, one has to deal with a time-based sequence of amplitudes. The following subsections briefly review some existing direct time-domain, lossless, and lossy techniques for the compression task. For an overview of data compression techniques, the reader is referred to [5], [9].

\section{A. Direct Time-Domain Approaches}

There are some simple approaches that are made up by some operations directly on the time domain of the acquired signal. These methods are often used in heartbeat detection and counting, achieving good compression ratios but failing in the perfect reconstruction of the signals, introducing distortion to the ECG signal. Among these operations, we have: amplitude scaling, Differential Pulse Code Modulation (DPCM), Amplitude Zone Time Epoch Coding (AZTEC), Turning Point (TP), and Coordinate Reduction Time Encoding Scheme (CORTES) [4], [7].

\section{B. Lossless Encoding Approaches}

The lossless encoding techniques can be classified in two major groups [9]: entropy coding and dictionary-based

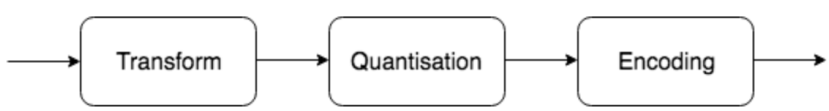

Fig. 3. The lossy encoding process block diagram with its three main operations [5].

coding. The lossless compression is achieved by removing the redundancy often found in the data. Among these techniques, we have for instance the entropy-based Huffman coding [3] and the dictionary-based Lempel-Ziv-Welch (LZW) [11]. The DEFLATE compression algorithm is based on the Lempel-Ziv 77 (LZ77) algorithm, for duplicate string elimination, followed by Huffman coding, for bit reduction. For each data block, the LZ77 algorithm finds repeated substrings and replaces the next occurrence of that substring by a pointer to the previous substring, with a pair of coordinates distance and length. The original sequences and the match lengths are compressed with one Huffman tree and the match distances are compressed with another tree. The built Huffman trees are encoded to go along with the rest of the data, so the receiver does not need to build the Huffman tree to decode the data. The Linear Predictive Coding (LPC) [5] is a type of predictive encoding scheme adequate for audio and speech signals. This type of coding is characterized by being an Auto-Regressive (AR) model, that is, a sample is linearly dependent on the previous samples. The main idea of LPC is the transmission of an error $e[n]$, which results from the subtraction between the original signal $o[n]$ and the predicted signal $p[n]$. The LPC coefficients are computed from the autocorrelation of the input signal and the number of coefficients of the predictor is set based on the desired amount of error between $o[n]$ and $p[n]$.

\section{Lossy Encoding Approaches}

Transformation-based methods [5] are the most used techniques to perform lossy encoding of audio and image data. The transformation methods are lossless being applied to enable better coefficient quantization, introducing loss, which results in a lower quality output with high compression ratio. These techniques consist in discarding less significant information, on the quantization stage, which tends to be irrelevant to the human perception of the signal. Figure 3 depicts the lossy encoding process with its three key stages. There are different approaches for the three building blocks of Figure 3. In this study, we address two well-known techniques for the transform operation, namely the Discrete Cosine Transform (DCT) and the Discrete Wavelet Transform (DWT) [5]. The DCT performs the representation of the input signal (a sequence of $N$ points) on a cosine basis functions. The DCT method is used in various applications such as in the lossy compression of audio signals, in the MP3 format. The DCT coefficients, $F[u], u \in\{0,1, \ldots, N-1\}$ for a one-dimensional $\mathrm{N}$-point signal are computed by

$$
F[u]=a[u] \sum_{n=0}^{N-1} f[n] \cos \left(\frac{\pi(2 n+1) u}{2 N}\right),
$$



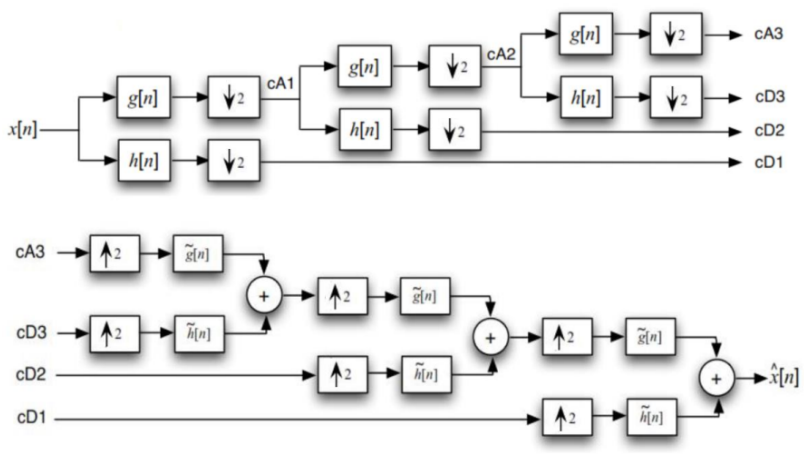

Fig. 4. The Discrete Wavelet Transform (DWT) [5]: top - the forward transform; bottom - the inverse transform.

where $f[n]$ is the input signal and $a[u]$ is a scaling factor computed as

$$
a[u]=\left\{\begin{array}{cc}
\sqrt{\frac{1}{N}}, & u=0 \\
\sqrt{\frac{2}{N}}, & u \in\{1, \ldots, N-1\} .
\end{array}\right.
$$

The reconstructed signal is given by

$$
f[n]=\sum_{u=0}^{N-1} a[u] F[u] \cos \left(\frac{\pi(2 u+1) n}{2 N}\right) .
$$

The DWT performs a decomposition of the input signal, depicted in the top part of Figure 4, through a Low-PassFilter (LPF) and a High-Pass-Filter (HPF), with impulse responses $g[n]$ and $h[n]$, respectively. The LPF filtering generates approximation coefficients (cA) and the HPF generates the detail coefficients. The filtering operations are followed by a dyadic decimation operation, usually called downsampling. The DWT provides a multi-resolution representation of signals, with information in time and frequency domains. As a result, the DWT decomposes a digital signal into different sub-bands so that the lower frequency subbands will have a finer frequency resolution and a coarser time resolution compared to the higher frequency sub-bands. The inverse DWT is represented in the bottom part of Figure 4, with the upsampling procedure with a factor of 2, applied before each filtering operation.

\section{EXPERIMENTAL EVALUATION}

In this section, we perform a comparison of different lossless and lossy approaches for the ECG compression task. We present a description of the evaluation procedure and evaluation metrics and then we move on to the experimental results attained with the techniques described in Section III, for ECG signals. We have considered a dataset provided by the Swedish National Road and Transport Research Institute contains signals from 18 different people, including ECG and SWA, for the same car and track, in both awake and drowsy states.

\section{A. Evaluation Metrics and Procedure}

The Compression Ratio (CR) is one of the most used metrics in signal compression and measures the data reduction achieved by a given compression method. When testing a method, it is intended to obtain high CR while maintaining acceptable signal quality. For the original, $o[n]$ and the compressed, $c[n]$, signals, the $\mathrm{CR}$ is given by

$$
\mathrm{CR}(o[n], c[n])=\frac{l_{o}}{l_{c}}: 1,
$$

where $l_{c}$ and $l_{o}$ are the length of the compressed and the original signal, respectively. For instance, a compression with a reduction to half the original size is represented as 2:1.

The Root-Mean-Squared Error (RMSE) is one of the most used distortion metrics. It represents how far the output samples are from the input and it is calculated by the squared root of the summation of the mean of the squared differences between the original signal $o[n]$ and the decoded signal $d[n]$,

$$
\operatorname{RMSE}(o[n], d[n])=\sqrt{\frac{1}{N} \sum_{n=0}^{N-1}(o[n]-d[n])^{2}} .
$$

The Signal-to-Noise Ratio (SNR) measures the quality of a signal affected by noise. In this case, the noise is given by the distortion introduced by the lossy encoding process on the quantization stage. SNR, expressed in $\mathrm{dB}$, is defined as

$$
\operatorname{SNR}(o[n], d[n])=10 \log _{10}\left(\frac{P_{o}}{P_{n}}\right),
$$

where $P_{o}$ and $P_{n}$ are the power of the original and noise/error signals, respectively. The use of SNR to denote the quality of the uniform quantization procedure leads to the SNRquantization (SNRQ) expression,

$$
\mathrm{SNRQ}=6.02 R+10 \log _{10}\left(\frac{3 P}{V^{2}}\right),
$$

where $R$ denotes the number of bits per sample, $P$ is the power of the quantized signal and $V$ is the maximum amplitude of the quantizer. SNRQ serves as the basis for comparing the attained transmission SNR (SNRt), after lossy encoding. Considering that the number of bits per symbol after quantisation is $R=12$, the supply voltage of the microcontroller $V=3.3$, and the power of the signal $P$ is roughly its squared amplitude $A^{2}$, then $\mathrm{SNRQ}=111.93 \mathrm{~dB}$, for the considered ECG signals.

The experimental procedure was conducted as follows. The algorithms were written in Matlab and developed to test source and transform-based compression methods. The performance of these methods were assessed with different metrics, described above, with the goal to identify the method that best suits the application of fatigue and drowsiness detection signals, which is the key goal of this module on the overall system [2]. We have considered a set of methods as described in the following subsection.

\section{B. Lossy and Lossless Encoding Techniques}

The following encoding methods were considered:

- DPCM + Huffman - The DPCM technique obtains values on the 8 -bit range of values $\{-128, \ldots, 127\}$, turning the signal quantized with 8 bits instead of 12. The Huffman code produces variable length codewords, depending on the probability of occurrence of 
the corresponding symbol. Thus, we perform a 12 to 8-bit compression before Huffman coding, so the output of it could fit in a Bluetooth Low Energy (BLE) frame. This results in a CR of 1.5:1 with an infinite SNRt (lossless technique). In case any DPCM sample is outside the 8-bit range, the samples are truncated at that limit to be sent via BLE, introducing error in the transmitted signal.

- $\mathbf{L P C}+\mathbf{L Z W}$ - The LPC model is computed from the mean of the first $a+1$ samples of three ECG signals. The initial coefficients of the LPC predictor both at the transmitter and receiver are the mean of the coefficients computed for each ECG signal. The LZW algorithm is applied to the low entropy error from the LPC encoder. To transmit in 8-bit frames of the BLE, the LZW dictionary has a maximum of 256 entries. The initial dictionary size, depends on the entropy of the error; an initial dictionary with 32 entries is adequate to handle the prediction for new ECG signals.

- DCT-based - The DCT can achieve a good performance in signal compression if there are no concerns with the signal amplitude, but only with sample reduction. The output signal has high amplitudes at the beginning, however, from the 4800 coefficient and beyond there are no significant amplitude variations, and from sample 11000 until the end, the amplitude can be discarded. Considering the first 11000 samples, this method can achieve a CR of 5.36:1, and the reconstructed signal has a SNRt of $54.94 \mathrm{~dB}$.

- DWT-based - The compression in the DWT is achieved by only transmitting the approximation coefficients while the detail coefficients are discarded due to their low amplitude. We applied the amplitude scaling, from 12 to 8 bits before applying the DWT with Daubechies wavelets (the db10 wavelet), since the ECG signal has a lot of vanishing moments and these wavelets are known to be adequate to represent signals with vanishing moments.

Table I shows the experimental results of these techniques, regarding the CR, RMSE, and SNRt measures.

TABLE I

EXPERIMENTAL RESULTS ON COMPRESSION AND DISTORTION

\begin{tabular}{|c|c|c|c|}
\hline Method & CR & RMSE & SNRt \\
\hline DPCM + Huffman & $1.5: 1$ & 0 & $\infty$ \\
\hline LPC (10 coefficients) + LZW & $4.57: 1$ & 0 & $\infty$ \\
\hline LPC + DEFLATE & $3.45: 1$ & 0 & $\infty$ \\
\hline \hline DCT-based & $5.36: 1$ & 0.33 & 54.94 \\
\hline Amplitude Scaling + DWT & $5.99: 1$ & 3.56 & 34.26 \\
\hline
\end{tabular}

\section{Discussion of the experimental results}

With all the compression tests done, it is possible to compare them in order to establish the best solution for the ECG signal compression. According to the CR values obtained, the technique using Amplitude Scaling with DWT proved to be the one with higher compression and acceptable distortion.
However, this is a lossy technique and it introduces some distortion in the signal, that cannot be acceptable for precise analysis, like medical analysis. For lossless compression, the technique using LPC and LZW is the one with the best CR, taking into account that this algorithm needs some time to correct the prediction error and to be effectively a lossless method.

\section{CONCLUSIONS}

The monitoring of a persons ECG signal and its recording has many applications. In this paper, we address a system in which the ECG signal is extracted with dry-electrodes placed in a conductive leather covering the steering wheel that can sense the electrical signal caused by the heartbeat of the person while having the hands on the wheel.

The amount of data acquired with this system undergoes a compression stage for transmission with the goal of reducing the necessary bandwidth. For this purpose, in this paper we have evaluated different lossy and lossless techniques for data compression. We have compared different techniques with standard metrics, using Matlab implementations. The method that achieved better compression is the lossy hybrid method using Amplitude Scaling and DWT with a CR of 5.99:1 with an acceptable distortion. However, the LPC+LZW method attains a CR of 4.57:1. The DCT-based method offers the second best CR and RMSE values with the highest SNRt, and thus it seems the best trade-off option. The choice of which method best suits varies with the type of application and for ECG pattern recognition it is preferable to preserve the signal than to reduce the amount of data.

As future work, we intend to fine tune the parameters of the best performing lossless and lossy techniques, for our ongoing development of the system to monitor drivers fatigue and drowsiness, to alert the driver from potential hazard.

\section{REFERENCES}

[1] C. Carreiras, A. Lourenço, H. Silva, and A. Fred. Comparative study of medical-grade and off-the-person ECG systems. In Proc International Workshop on Pervasive Electrocardiography - IWoPE, 2013.

[2] A. Cerca. Fatigue and drowsiness detection using inertial sensors and electrocardiogram, Instituto Superior de Engenharia de Lisboa, MSc Thesis, 2018.

[3] D. Huffman. A method for the construction of minimum-redundancy codes. Proceedings of the Institute of Electrical and Radio Engineers, 40(9):1098-1101, September 1952.

[4] S. Jalaledinne, C. Hutchens, R. Strattan, and W. Coberly. ECG data compression techniques - a unified approach. IEEE, 1990.

[5] Z. Li, M. Drew, and J. Liu. Fundamentals of Multimedia, 2nd edition. Springer International Publishing, 2014.

[6] A. Lourenço, A. Alves, C. Carreiras, R. Duarte, and A. Fred. Cardiowheel: ECG biometrics on the steering wheel. In ECML/PKDD, September 2015.

[7] Priyanka and I. Saini. Analysis ECG data compression techniques - a survey approach. International Journal of Emerging Technology and Advanced Engineering, 2013.

[8] C. Saritha, V. Sukanya, and Y. Murthy. ECG signal analysis using wavelet transforms. Bulg. J. Phys., 35, pages 68-77, 2008.

[9] K. Sayood. Introduction to Data Compression, 5th edition. Morgan Kaufmann Publishers, 2017.

[10] U. Tiwary and B. Gohel. Automated risk identification of myocardial infarction using relative frequency band and coefficient features from ECG. The Open Biomedical Engineering Journal, 4, 2010.

[11] T. Welch. A technique for high-performance data compression. IEEE Computer, 17(6):8-19, 1984. 